Analytical Methods

\title{
Phenolic profiling of the skin, pulp and seeds of Albariño grapes using hybrid quadrupole time-of-flight and triple-quadrupole mass spectrometry
}

\author{
Giuseppe Di Lecce ${ }^{\mathrm{a}}$, Sara Arranz ${ }^{\mathrm{b}, \mathrm{d}}$, Olga Jáuregui ${ }^{\mathrm{c}}$, Anna Tresserra-Rimbau ${ }^{\mathrm{a}, \mathrm{d}}$, Paola Quifer-Rada ${ }^{\mathrm{a}, \mathrm{d}}$, \\ Rosa M. Lamuela-Raventós ${ }^{\text {a,d,* }}$ \\ ${ }^{a}$ Nutrition and Food Science Department, XaRTA, INSA, Pharmacy School, University of Barcelona, Barcelona, Spain \\ ${ }^{\mathrm{b}}$ Department of Internal Medicine, Hospital Clinic, Institut d'Investigacions Biomédiques August Pi i Sunyer (IDIBAPS), University of Barcelona, Barcelona, Spain \\ ${ }^{\mathrm{c}}$ Unitat de Tècniques Separatives, Centres Cientifics i Tecnologics (CCiTUB), Universitat de Barcelona, Josep Samitier 1-5, 08028 Barcelona, Spain \\ ${ }^{\mathrm{d}}$ CIBER CB06/03 Fisiopatología de la Obesidad y la Nutrición, (CIBEROBN), and RETICS RD06/0045/0003, Instituto de Salud Carlos III, Spain
}

\section{A R T I C L E I N F O}

\section{Article history:}

Received 17 November 2011

Received in revised form 27 July 2012

Accepted 28 August 2013

Available online 4 September 2013

\section{Keywords:}

Albariño grape

Phenolic compounds

Hybrid quadrupole time of flight

Flavanol hexose

Accurate mass

Neutral loss scan

\begin{abstract}
A B S T R A C T
This paper describes for the first time a complete characterisation of the phenolic compounds in different anatomical parts of the Albariño grape. The application of high-performance liquid chromatography coupled with two complementary techniques, hybrid quadrupole time-of-flight and triple-quadrupole mass spectrometry, allowed the phenolic composition of the Albariño grape to be unambiguously identified and quantified. A more complete phenolic profile was obtained by product ion and precursor ion scans, while a neutral loss scan at $152 \mathrm{u}$ enabled a fast screening of procyanidin dimers, trimers and their galloylated derivatives. The compounds were confirmed by accurate mass measurements in QqToF-MS and QqToF-MS/MS modes at high resolution, and good fits were obtained for all investigated ions, with errors ranging from 0.2 to $4.5 \mathrm{mDa}$. To the best of our knowledge, two flavanol monomer hexosides were detected in the grape berry for the first time.
\end{abstract}

() 2013 Elsevier Ltd. All rights reserved.

\section{Introduction}

Albariño (Vitis vinifera L.) is the most important white grape variety grown in the northwest of Spain (Galicia), notably in the Rías Baixas Denomination of Origin. Although there are five different varieties of grape cultived in this region, Albariño grape provide $95 \%$ of the annual harvest obtaining a total of 40 million $\mathrm{kg}$ of grapes to produce 286,000 hectoliters of wine annually. Albariño wine is characterised by an appreciated aromatic profile and organoleptic properties (Diéguez, Lois, Gómez, \& de la Peña, 2003; Vilanova, Genisheva, Masa, \& Oliveira, 2010). Masa, Vilanova, and Pomar (2007) and Rodríguez-Bernaldo de Quirós, Lage-Yusty, and López-Hernández (2009) determined the flavonoid profile of Albariño grape skin and the antioxidant activity of Albariño wines by high performance liquid chromatography (HPLC) (Masa et al., 2007; Rodríguez-Bernaldo de Quirós et al., 2009).

Phenolic compounds are responsible for the colour, astringency and bitterness of wines and it has been demonstrated that the sen-

\footnotetext{
* Corresponding author. Address: Nutrition and Food Science Department, XaRTA, INSA, Pharmacy School, University of Barcelona, Av. Joan XXIII s/n, 08028 Barcelona, Spain. Tel.: +34 934034843; fax: +34 934035931.

E-mail address: lamuela@ub.edu (R.M. Lamuela-Raventós).
}

sory perception of coarseness increases with the degree of galloylation of proantocyanidins (Vidal et al., 2003).

Grape phenols consist of a wide range of structures diversely distributed in every part of the berry (Adam, 2006), but they are present mainly in the skin and seed (Rodríguez Montealegre, Romero Peces, Chacón Vozmediano, Martínez Gascueña, \& García Romero, 2006; Dietmar, Achim, Reinhold, \& Schieber, 2004).

The most abundant phenolic compounds in white grape skin are flavonols, while flavan-3-ol monomers such as (+)-catechin and (-)-epicatechin, as well as dimers, trimers and polymeric forms, also called procyanidins (2-10 units), are present mainly in grape seed. These compounds may contain subunits of gallic acid, epigallocatechin or epicatechin gallate linked by an interflavan bond (Hayasaka, Waters, Cheynier, Herderich, \& Vidal, 2003).

In last two decades liquid chromatography mass spectrometry has been widely employed for the characterisation of several food matrices (Justesen, Knuthsen, \& Leth, 1998; Zhou, Xu, \& Choi, 2009). Electrospray ionisation has proven to be a powerful tool that facilitates the analysis of non-volatile, thermally labile compounds. Different mass analysers, triple-quadrupole instruments (SánchezRabaneda et al., 2004; Cavaliere et al., 2008), ion-trap mass analysers, and high-resolution instruments such as time-of-flight (or the hybrid configuration quadrupole-time-of-flight, Vallverdú-Queralt, 
Jáuregui, Di Lecce, Andrés-Lacueva \& Lamuela-Raventós, 2011b) and Fourier trasformation mass spectrometry (FTMS, VallverduQueralt, Jáuregui, Medina-Remón, Andrés-Lacueva, \& LamuelaRaventós, 2010) have been used for chemical characterisation of food matrices.

Specifically, polyphenol composition in food has been analysed using triple quadrupole instruments, applying MS/MS techniques such as product ion scan (PIS), precursor ion scan (PrI), and neutral loss scan (NL) (Sánchez-Rabaneda et al., 2004; Vallverdu-Queralt et al., 2010). A quadrupole instrument in full scan mode shows a poor signal-to-noise-ratio (if compared with the ratio of a high resolution instrument) but MS/MS techniques such as PrI or NL allow polyphenol families to be screened. In addition, hybrid high-resolution instruments such as Qq-ToF and IT-FTMS can produce high-quality MS/MS spectra, including high-resolution data for the determination of molecular formulae. Both MS and MS/MS experiments can be performed for high accuracy and high resolution analysis.

As far as we know, recent studies of the phenolic profile of the Albariño cultivar have only described the flavonoid composition. The aim of this paper is to report the first study on the qualitative and quantitative characterisation of phenolics in the different anatomical parts of the Albariño grape using two complementary QqToF and QqQ instruments to determine structures based on fragmentation patterns.

\section{Materials and methods}

\subsection{Chemicals}

The standards were handled without exposure to light. Vanillic acid, 4-hydroxybenzoic acid, 3-hydroxybenzoic acid, trans-caffeic acid, trans-ferulic acid, protocatechuic acid, $m-, o$ - and $p$-coumaric acids, gallic acid, homovanillic acid, (+)-catechin, (-)-epicatechin, trans-resveratrol, trans-piceid, kaempferol, myricetin, apigenin, (-)-epigallocatechin, (+)-catechin-gallate, quercetin, quercetin-3$O$-rutinoside, quercetin-3-O-glucuronide, quercetin-3-O-glucoside, isorhamnetin-3-O-glucoside and L-tryptophan were purchased from Sigma-Aldrich (St Louis, MO, USA). Kaempferol-3-O-glucoside, procyanidin dimers A2, B1 and B2, trimer C1, (-)-epicatechingallate, ethyl gallate, catechin-gallate and tyrosol were purchased from Extrasynthèese (Genay, France). cis-Resveratrol and cis-piceid were obtained after exposure of the trans-isomer standards to UV light (Romero-Pérez, Ibern-Gómes, Lamuela-Raventós, \& de la Torre-Boronat, 1999), whereas trans-caftaric and trans-coutaric acids were isolated from grapes (Vrhovšek, 1998). HPLC-grade acetonitrile and acetic acid were purchased from Scharlau Chemie S.A. (Barcelona, Spain), while ultrapure water was obtained from a Millipore system (Millipore, Bedford, MA, USA).

\subsection{Extraction procedure}

Albariño grape berries were harvested at the Miguel Torres winery in Vilafranca del Penedès (Barcelona, Spain). The samples were collected at $22 \pm 0.6$ Brix and immediately frozen $\left(-20^{\circ} \mathrm{C}\right)$ until analysis. Sample extraction was performed in a dark room with a red safety light to prevent oxidation of the analytes during the process. Frozen grapes were manually separated into skin $(0.5 \mathrm{~g})$, pulp $(5 \mathrm{~g})$ and seeds $(0.5 \mathrm{~g})$. The extraction procedure, for the three fractions, was carried out under low temperature, as previously described by our research group (Vallverdú-Queralt et al., 2011a), using $5 \mathrm{~mL}$ of ethanol/water 80:20 (v/v) at pH 3.5 (acetic acid). The homogenates, obtained by an Ultra Turrax (IKA, Staufen, Germany), were centrifuged $\left(2500 \mathrm{~g}, 20 \mathrm{~min}\right.$ at $\left.4{ }^{\circ} \mathrm{C}\right)$, and the supernatants were collected; the extraction procedure was repeated two times. Both fractions were combined and the ethanolic portion was evaporated with a sample concentrator (Techne, Duxford, Cambridge, UK) at room temperature under a stream of nitrogen gas. After filtration of the aqueous extracts with $0.45 \mu \mathrm{m}$ PTFE syringe filters (Waters Corporation, Massachusetts, USA), the samples were stored at $-20^{\circ} \mathrm{C}$ and then injected into the HPLC-UV-QqToF and QqQ systems.

\subsection{HPLC-UV and mass spectrometry conditions}

The chromatography was performed with an HPLC Agilent 1200 RRLC (Santa Clara, CA, USA), using a Nucleosil 120 C18 column $(250 \mathrm{~mm} \times 4 \mathrm{~mm}, 5 \mu \mathrm{m}$ particle size, Teknokroma, Barcelona, Spain). A constant flow rate of $0.8 \mathrm{~mL} \mathrm{~min}^{-1}$ was used with two solvents: solvent A consisted of water with $0.8 \%$ acetic acid $(\mathrm{pH}$ 2.65 ), and solvent B was $20 \%$ solvent A mixed with $80 \%$ acetonitrile; injection volume was $20 \mu \mathrm{L}$. The column was kept at $40^{\circ} \mathrm{C}$ and the separation of phenolic compounds was carried out in $45 \mathrm{~min}$ under the following conditions: $0 \mathrm{~min}, 100 \% \mathrm{~A} ; 5 \mathrm{~min}$, $98 \% \mathrm{~A} ; 10 \mathrm{~min}, 96 \% \mathrm{~A} ; 15 \mathrm{~min}, 90 \% \mathrm{~A} ; 30 \mathrm{~min}, 80 \% \mathrm{~A} ; 35 \mathrm{~min}$, $70 \%$ A; 40 min 0\% A and 45 min, 100\% A (Betés-Saura, Andrés-Lacueva, \& Lamuela-Raventós, 1996). The column was equilibrated for 5 min prior to each analysis. The chromatograms were monitored, with UV detector Agilent SL Plus, at three wavelengths: 280, 320, and $365 \mathrm{~nm}$. Each wavelength was suitable for each group of compounds: $280 \mathrm{~nm}$ was used for hydroxybenzoic acids, flavan-3-ols and the oligomeric procyanidins, $320 \mathrm{~nm}$ for hydroxycinnamic acids and their tartaric esters, and $365 \mathrm{~nm}$ for flavonols.

Individual compounds were quantified using a calibration curve of the corresponding standard compound. When reference compounds were not available, the calibration of structurally related substances was used. All analysis were performed in triplicate.

\subsubsection{QqToF analysis}

The HPLC system was coupled on-line to a hybrid quadrupole time-of-flight QSTAR Elite (ABSciex, Concord, Ontario, Canada). The MS acquisition was performed using negative ionisation between $\mathrm{m} / \mathrm{z} 100$ and 1050 with the Turbo Ionspray source. In addition, QqToF was used to obtain product ion information. The MS parameters were: ion spray voltage, -4200 ; declustering potential (DP), -60; focusing potential (FP), 190; declustering potential two (DP2), 15; ion release delay (IRD), $6 \mathrm{~V}$; ion release width (IRW), 5 ms; nebulizer gas, 50 (arbitrary units), curtain gas, 60 (arbitrary units), and auxiliary gas $\mathrm{N}_{2}, 6000 \mathrm{~cm}^{3} \mathrm{~min}^{-1}$ heated at $400^{\circ} \mathrm{C}$. The QqToF-MS instrument was calibrated after every three samples injected using two external reference compounds at $\mathrm{m} / \mathrm{z}$ $112.9854\left(\mathrm{CF}_{3} \mathrm{COO}^{-}\right)$and $\mathrm{m} / z 1033.9880\left(\mathrm{P}_{3} \mathrm{~N}_{3}\left(\mathrm{OCH}_{2}\left(\mathrm{CF}_{2}\right) \mathrm{CF}_{2} \mathrm{H}\right)_{6-}\right.$ $\mathrm{CF}_{3} \mathrm{COO}^{-}$), respectively (1 pmol $\mu \mathrm{L}^{-1}$, ESI Tuning Mix Agilent solution). The MS/MS acquisition was also performed using information-dependent acquisition (IDA) between $\mathrm{m} / \mathrm{z} 100$ and 1050. IDA experiments were done at a fixed collision energy of $30 \mathrm{~V}$ and modified if no-fragmentation (or excessive) was produced. Acquisition and analysis of data were performed with Analyst QS 2.0 software (ABSciex, Concord, Ontario, Canada).

\subsubsection{QqQ analysis}

An API 3000 triple quadrupole mass spectrometer (ABSciex, Concord, Ontario, Canada) equipped with a Turbo Ionspray source in negative-ion mode was used to obtain product ion and neutral loss information. Turbo Ionspray source settings were as follows: ion spray voltage, $-3500 \mathrm{~V}$; nebulizer gas, 10 (arbitrary units); curtain gas, 12 (arbitrary units); collision gas, 4 (arbitrary units); focusing potential, $-200 \mathrm{~V}$; entrance potential, $10 \mathrm{~V}$; drying gas $\left(\mathrm{N}_{2}\right)$, heated to $400{ }^{\circ} \mathrm{C}$ and introduced at a flow rate of $8000 \mathrm{~mL} \mathrm{~min}^{-1}$. The DP and collision energy (CE) were optimised for (+)-catechin (DP -50 and $C E-25 \mathrm{~V}$ ), procyanidin B1 (DP -50 
and $C E-35 \mathrm{~V})$, trans-caffeic acid (DP -40 and $C E-20 \mathrm{~V}$ ), and quercetin-3-O-glucoside (DP -60 and $C E-30 \mathrm{~V}$ ) in infusion experiments. Individual standard solutions $\left(10 \mu \mathrm{g} \mathrm{mL}^{-1}\right)$ dissolved in 50:50 (v/v) mobile phase were infused at a constant flow rate of $5 \mu \mathrm{min}^{-1}$, using a syringe pump (Harvard Apparatus, Holliston, MA, USA). Data acquisition was performed scanning from $\mathrm{m} / \mathrm{z}$ 100 to 1050 in profile mode and using a cycle time of $2 \mathrm{~s}$ with a step size of $0.1 \mathrm{u}$ and a pause between each scan of $2 \mathrm{~ms}$. In NL experiment, loss of $162 \mathrm{u}$ corresponds to the loss of a glucose or galactose, while loss of $152 \mathrm{u}$, derived from the product of RetroDiels-Alder rearrangement, correspond to dimer and trimer procyanidins as well as flavanol galloyl derivatives. Neutral loss experiments at $162 \mathrm{u}$ and $152 \mathrm{u}$, were performed by scanning within the range of 300-600 $\mathrm{u}$ and from 250-900 u, respectively.

\section{Results and discussion}

\subsection{HPLC-ESI-QqToF-MS and HPLC-ESI-QqQ-MS for the} determination of phenolic compounds in grape skin, pulp and seed extracts

Phenolic extracts of skin, pulp and seed of Albariño grapes were analysed with two complementary QqToF and QqQ instruments to determine structures based on fragmentation patterns, using QqToF in PIS mode and QqQ in PrI and NL mode. In addition, information-dependent acquisition (IDA) by QqToF was used to generate a peak list of ions present in the spectrum at the time of analysis; this peak list was subjected to a series of user-defined criteria to select precursor ions of interest based on filters such as intensity threshold, charge state, isotope pattern and others. In general, we observed the deprotonated molecule $[\mathrm{M}-\mathrm{H}]^{-}$and its characteristic product ions by MS/MS experiments. The 43 compounds are depicted in Fig. 1 and listed in Table 1 along with their retention time, molecular formulae and $\mathrm{mDa}$ of error between the experimental mass and the theoretical mass of each phenol investigated.

Thus, bearing in mind the importance of phenolic compounds as taxonomical markers (Vallverdu-Queralt et al., 2010), a precise characterisation of Albariño grape was obtained.

\subsubsection{Hydroxybenzoic acid and its derivatives}

Gallic acid ( $m / z 169)$ was the first compound to elute in skin and seed extract chromatograms. The product ion scan of the deprotonated molecule $[\mathrm{M}-\mathrm{H}]^{-}$showed the typical loss of $\mathrm{CO}_{2}$, giving an ion at $m / z 125$ [M-H-44] $]^{-}$as the characteristic fragment. This compound was confirmed by comparison with the calculated mass error $(0.9 \mathrm{mDa})$ and reference compound.

LC-QqToF-MS analysis of seeds showed ions at $\mathrm{m} / \mathrm{z} 331$ and 315 (peaks 2,14 and 4,10 , respectively), which were tentatively identified as deprotonated molecules of isomers of gallic acid hexose and protocatechuic acid hexose, respectively. Product ion scan of both ions showed the loss of hexose [M-H-162 $]^{-}$, followed by the loss of $\mathrm{CO}_{2}$ [M-H-162-44] $]^{-}$. It was not possible to differentiate between the isomers on the basis of fragments and relative intensities in MS/MS spectra in PIS mode (Table 1 ).

Gallic acid dihexose ( $m / z$ 493) was also tentatively identified in seed and skin extracts: the PIS of the deprotonated molecule $(\mathrm{m} / \mathrm{z}$ 493) showed two ions at $\mathrm{m} / \mathrm{z} 331$ and 169 derived from the loss of one and two hexose units, respectively. Furthermore, a very low mass error $(0.3 \mathrm{mDa})$ was obtained with the QqToF instrument. The identification hypothesis was strengthened by the information obtained with the QqQ instrument, through neutral loss scan of $162 \mathrm{u}$ and precursor ion scan of $m / z 169$.

\subsubsection{Hydroxycinnamic acid and its derivatives}

The skin extract revealed the presence of $p$-coumaric acid $(\mathrm{m} / \mathrm{z}$ 163), which was corroborated by product ion scan experiment showing a predominant ion at $\mathrm{m} / z 119\left(\right.$ loss of $\left.\mathrm{CO}_{2}\right)$. The presence of coumaric acid hexose $(\mathrm{m} / z$ 325) was also detected in skin and pulp. The PIS of this ion showed a characteristic fragmentation involving cleavage of the intact sugar $[\mathrm{M}-\mathrm{H}-162]^{-}(\mathrm{m} / z$ 163), and an ion corresponding to the loss of a methyl $\left(\mathrm{m} / z\right.$ 148) and $\mathrm{CO}_{2}$ from aglycone $(m / z$ 119). Peak identification was accomplished by comparing MS/MS fragmentation with reported data obtained by LC-ESI-MS in negative mode (Vallverdu-Queralt et al., 2010). NL of $162 \mathrm{u}$ and PrI of $m / z 163$ by QqQ were useful for providing an unequivocal identification of hydroxycinnamic hexose.

\subsubsection{Hydroxycinnamic tartaric esters}

The skin and pulp extract chromatograms showed two ions at $\mathrm{m} / \mathrm{z} 311$ (peaks 7 and 8), two ions at $\mathrm{m} / \mathrm{z} 295$ (peaks 15 and 17), and one ion at $m / z 325$ (peak 21). These deprotonated molecules $[\mathrm{M}-\mathrm{H}]^{-}$were tentatively identified as hydroxycinnamic acid tartaric esters. The ions at $\mathrm{m} / \mathrm{z} 311$ were identified as cis and trans-caffeoyl tartaric acid (caftaric acid); their PIS revealed a fragment at $\mathrm{m} / \mathrm{z} 179$ corresponding to caffeic acid, after the cleavage of the ester bond, and a low intensity signal at $m / z 135$ was ascribed to decarboxylated caffeic acid. MS/MS data were corroborated by comparison with reference compounds isolated from grape pomace (Vrhovšek, 1998). The ions at $m / z 295$ showed a fragmentation pattern similar to caftaric acid and were identified as cis and trans-coumaroil tartaric acid (coutaric acid) after comparison with the reference compound. The PIS produced only one ion fragment at $m / z 163$, which was ascribed to coumaric acid. In contrast, the deprotonated molecule at $\mathrm{m} / \mathrm{z} 325$ showed a fragment at $m / z 193$ attributed to ferulic acid, and an ion at $m / z$ 149 that indicated a loss of $\mathrm{CO}_{2}$ from the free ferulic acid. It was not possible identify the isomeric configuration of feruloyl tartaric acid, otherwise known as fertaric acid. The PrI at $m / z$ 179, 163 and 193 confirmed the presence of the described peaks. Only cis and trans-caftaric acid and fertaric acid were found in the pulp extract. Among the aforementioned compounds, transcaftaric acid is considered a major substance for coupled oxidation and enzymatic browning reactions in grape processing (Kroon \& Williamson, 1999).

\subsubsection{Flavan-3-ols}

Reverse phase HPLC procedures provided a good baseline resolution for the flavan-3-ols, which consisted of (+)-catechin, (-)-epicatechin, their condensed product and corresponding galloylated derivatives that exhibited monomeric, dimeric and trimeric degrees of polymerisation. When the degree of polymerisation increased, the procyanidins were eluted as a single peak at the end of the chromatogram (Fig. 1). The resolved procyanidins present in Albariño seed, skin and pulp are mainly dimers $(\mathrm{m} / \mathrm{z} 577)$ and trimers $(m / z 865)$ in which the elemental units are linked by $C 4$ C8 interflavan bonds (B-type). Structural variations in procyanidin oligomers may also occur with the formation of a second interflavanoid bond by $\mathrm{C}-\mathrm{O}$ oxidative coupling to form A-type oligomers. Due to the complexity of this conversion, A-type procyanidins are not as frequently encountered in nature as B-type oligomers. Procyanidin A-type linkage shows a different fragmentation pathway than B-type linkage (Flamini, 2003), and in Albariño grapes no procyanidin A-type linkages were observed.

Peaks 23 and $28(\mathrm{~m} / \mathrm{z} 289)$ were identified as (+)-catechin and (-)-epicatechin, respectively, after comparison with the authentic standard. Both flavan-3-ols were identified in the three different fractions, with mass errors below $0.7 \mathrm{mDa}$. Up to four procyanidin dimers $(m / z 577$, peaks $19,20,26$ and 27$)$ were identified in the seed extract. The PIS at $m / z 577$ showed a Retro-Diels-Alder 
(RDA) product with a neutral loss of $152 \mathrm{u}$ [M-H-152] $]^{-}$followed by loss of a water molecule $\left[\mathrm{M}-\mathrm{H}-152-\mathrm{H}_{2} \mathrm{O}\right]^{-}$. Other fragments at $\mathrm{m} / \mathrm{z}$ 289 and 245, derived from the interflavanic bond cleavage, were also observed (Table 1). Procyanidins B1 and B2 were corroborated by reference compounds, while the elution order of dimers B3 and B4 were assigned referring to the study by Monagas, Suárez, Gómez-Cordovés, and Bartolomé (2005).

LC-QqToF-MS analysis of the seed extract revealed four peaks $(6,24,25$ and 30$)$ at $m / z 865$. Product ion scan showed a base peak at $m / z 289$ and two minor ions at $m / z 577$ and 425 , which were also observed for the reference procyanidin C1. Additionally, an ion at $m / z 695$ was registered due to the RDA and successive loss of water $\left[\mathrm{M}-\mathrm{H}-152-\mathrm{H}_{2} \mathrm{O}\right]^{-}$. Peaks 24,25 and 30 were tentatively identified as procyanidin trimer isomers, but complete identification was not possible without standards.

Thus, on the basis of information obtained by PIS, when a NL experiment of $152 \mathrm{u}$ was conducted by QqQ the total ion current showed deprotonated molecules belonging to dimer $(\mathrm{m} / \mathrm{z} 577)$ and trimer procyanidins ( $m / z$ 865, Fig. 3). Various studies of oligomeric and polymeric procyanidins in grape seed extracts have proposed a fragmentation scheme of ions derived from B-type procyanidins (Zhao, Pang, \& Dixon, 2010; Sun \& Miller, 2003; Gu et al., 2003).

Waterhouse, Ignelzi, and Shirley (2000) demonstrated that in grape seeds, the single unresolved peak, at the end of the chromatogram, corresponds to a mixture of high molecular mass procyanidin polymers.
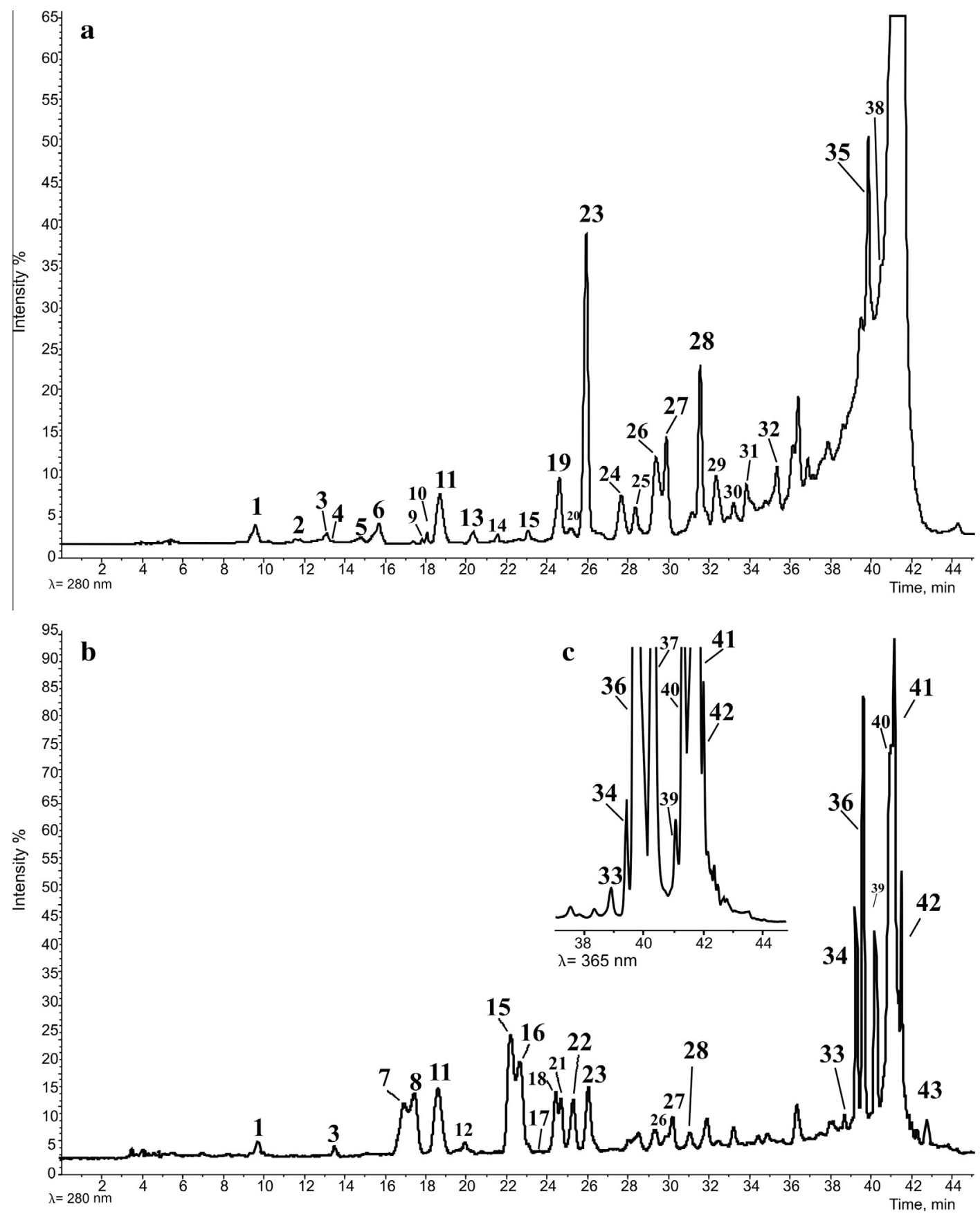

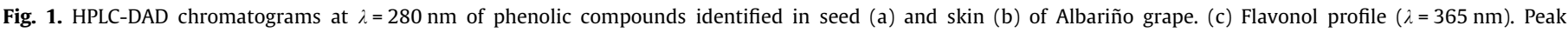
identification is shown in Table 1. 


\begin{tabular}{|c|c|c|c|c|c|c|c|c|c|c|c|}
\hline \multirow[t]{2}{*}{ Peaks } & \multirow{2}{*}{$\begin{array}{l}\text { Retention } \\
\text { time }\end{array}$} & \multirow[t]{2}{*}{ Compounds } & \multirow[t]{2}{*}{ Fractions } & \multirow[t]{2}{*}[\mathrm{M}-\mathrm{H}]{$^{-}$} & \multirow[t]{2}{*}{ Fragments $m / z$ (\% intensities) } & \multicolumn{3}{|c|}{ MS/MS experiments } & \multirow{2}{*}{$\begin{array}{l}\text { Theoretical } \\
\text { mass }\end{array}$} & \multirow{2}{*}{$\begin{array}{l}\text { Mass difference } \\
(\mathrm{mDa})^{\mathrm{a}}\end{array}$} & \multirow[t]{2}{*}{ Formula } \\
\hline & & & & & & $\begin{array}{l}\text { PIS (Qq- } \\
\text { ToF) }\end{array}$ & $\mathrm{NL}$ & $\begin{array}{l}\operatorname{PrI} \\
(\mathrm{QqQ})\end{array}$ & & & \\
\hline 1 & 9.35 & Gallic acid ${ }^{*}$ & se, sk & 169.0151 & $169(100), 125(25)$ & 169 & & & 169.0142 & 0.9 & $\mathrm{C}_{7} \mathrm{H}_{6} \mathrm{O}_{5}$ \\
\hline 2 & 11.82 & Gallic acid hexose I & se & 331.0700 & 331 (5), 169 (70), $125(100)$ & 331 & 162 & 169 & 331.0670 & 3.0 & $\mathrm{C}_{13} \mathrm{H}_{16} \mathrm{O}_{10}$ \\
\hline 3 & 13.08 & Gallic acid dihexose & se, sk & 493.1195 & 493 (9), $331(100), 169(100)$ & 493 & & 169 & 493.1198 & -0.3 & $\mathrm{C}_{19} \mathrm{H}_{26} \mathrm{O}_{15}$ \\
\hline 4 & 13.21 & Protocatechuic acid-O-hexoside & se & 315.0723 & $153(100), 109(40)$ & 315 & 162 & 153 & 315.0721 & 0.2 & $\mathrm{C}_{13} \mathrm{H}_{16} \mathrm{O}_{9}$ \\
\hline 5 & 14.76 & (epi)gallocatechin-(epi)catechin & se & 593.1332 & 423 (78), 305 (100), 289 (28) & 593 & & & 593.1300 & 3.2 & $\mathrm{C}_{30} \mathrm{H}_{26} \mathrm{O}_{13}$ \\
\hline 6 & 15.72 & Procyanidin trimer $\mathrm{C} 1$ & se & 865.1950 & $\begin{array}{l}865(37), 695 \text { (100), } 577 \text { (1), } 407 \text { (64), } 289 \\
(42)\end{array}$ & 865 & 152 & 289 & 865.1985 & -3.5 & $\mathrm{C}_{45} \mathrm{H}_{38} \mathrm{O}_{18}$ \\
\hline 7 & 16.90 & cis-caftaric acid & sk, pu & 311.0413 & $179(100), 135(54)$ & 311 & & 179 & 311.0418 & 0.5 & $\mathrm{C}_{13} \mathrm{H}_{12} \mathrm{O}_{9}$ \\
\hline 8 & 17.49 & trans-caftaric acid $^{*}$ & sk, pu & 311.0411 & 179 (100), 135 (40) & 311 & & 179 & 311.0414 & 0.3 & $\mathrm{C}_{13} \mathrm{H}_{12} \mathrm{O}_{9}$ \\
\hline 9 & 17.84 & (epi)gallocatechin-3-gallate & se & 457.0783 & $305(100), 169(65)$ & 457 & & & 457.0790 & 0.7 & $\mathrm{C}_{22} \mathrm{H}_{18} \mathrm{O}_{11}$ \\
\hline 10 & 18.01 & Protocatechuic acid- $O$-hexoside & se & 315.0751 & $153(100), 109(40)$ & 315 & 162 & 153 & 315.0781 & 3.0 & $\mathrm{C}_{13} \mathrm{H}_{16} \mathrm{O}_{9}$ \\
\hline 11 & 18.64 & L-tryptophan ${ }^{*}$ & se, sk, pu & 203.0847 & $142(9), 116(100)$ & 203 & & & 203.0868 & 2.1 & $\mathrm{C}_{11} \mathrm{H}_{12} \mathrm{~N}_{2} \mathrm{O}_{2}$ \\
\hline 12 & 19.82 & Epigallocatechin & sk & 305.0699 & 261 (100), $221(25), 179(34)$ & 305 & & & 305.0732 & 3.3 & $\mathrm{C}_{15} \mathrm{H}_{14} \mathrm{O}_{7}$ \\
\hline 13 & 20.23 & (epi)catechin-hexose & se & 451.1266 & $289(100), 245(40)$ & 451 & 162 & 289 & 451.1287 & 2.1 & $\mathrm{C}_{21} \mathrm{H}_{24} \mathrm{O}_{11}$ \\
\hline 14 & 21.56 & Gallic acid hexose II & se & 331.0700 & 331 (12), 169 (100), $125(84)$ & 331 & 162 & 169 & 331.0670 & 3.0 & $\mathrm{C}_{13} \mathrm{H}_{16} \mathrm{O}_{10}$ \\
\hline 15 & 22.35 & cis-coutaric acid & sk & 295.0469 & $163(32), 119(100)$ & 295 & & 163 & 295.0479 & 1.0 & $\mathrm{C}_{13} \mathrm{H}_{12} \mathrm{O}_{8}$ \\
\hline 16 & 22.83 & (epi)catechin-hexose & se & 451.1266 & 289 (100), $245(54)$ & 451 & 162 & 289 & 451.1287 & 2.1 & $\mathrm{C}_{21} \mathrm{H}_{24} \mathrm{O}_{11}$ \\
\hline 17 & 22.91 & trans-coutaric acid & sk & 295.0460 & $163(53), 119(100)$ & 295 & & 163 & 295.0461 & 0.1 & $\mathrm{C}_{13} \mathrm{H}_{12} \mathrm{O}_{8}$ \\
\hline 18 & 23.34 & Coumaric acid-O-hexoside & sk, pu & 325.0919 & $163(50), 148(30), 119(100)$ & 325 & 162 & 163 & 325.0928 & 0.9 & $\mathrm{C}_{15} \mathrm{H}_{18} \mathrm{O}_{8}$ \\
\hline 19 & 24.51 & Procyanidin B3 & se, pu & 577.1331 & 407 (75), $289(81), 245(67)$ & 577 & 152 & 289 & 577.1311 & -2.0 & $\mathrm{C}_{30} \mathrm{H}_{26} \mathrm{O}_{12}$ \\
\hline 20 & 24.75 & Procyanidin B1 ${ }^{*}$ & se & 577.1334 & 407 (75), 289 (70), $245(45)$ & 577 & 152 & 289 & 577.1317 & -1.7 & $\mathrm{C}_{30} \mathrm{H}_{26} \mathrm{O}_{12}$ \\
\hline 21 & 24.45 & Fertaric acid & sk, pu & 325.0585 & $193(100), 149(30)$ & 325 & & 193 & 325.0605 & 2.0 & $\mathrm{C}_{14} \mathrm{H}_{14} \mathrm{O}_{9}$ \\
\hline 22 & 24.82 & $p$-Coumaric acid ${ }^{*}$ & sk & 163.0418 & $163(20), 119(100)$ & 163 & & & 163.0436 & 1.8 & $\mathrm{C}_{9} \mathrm{H}_{8} \mathrm{O}_{3}$ \\
\hline 23 & 25.93 & $(+)$-Catechin & se, sk, pu & 289.0710 & 245(100), $205(65)$ & 289 & & & 289.0703 & -0.7 & $\mathrm{C}_{15} \mathrm{H}_{14} \mathrm{O}_{6}$ \\
\hline 24 & 27.72 & Procyanidin trimer I & se & 865.1954 & $\begin{array}{l}865 \text { (55), } 695 \text { (80), } 577 \text { (68), } 425 \text { (88), } 289 \\
(81)\end{array}$ & 865 & 152 & 289 & 865.1923 & -3.1 & $\mathrm{C}_{45} \mathrm{H}_{38} \mathrm{O}_{18}$ \\
\hline 25 & 28.30 & Procyanidin trimer II & se & 865.1971 & $\begin{array}{l}865(28), 695 \text { (19), } 577 \text { (33) } 575 \text { (21), } 289 \\
(100)\end{array}$ & 865 & 152 & 289 & 865.1957 & -1.4 & $\mathrm{C}_{45} \mathrm{H}_{38} \mathrm{O}_{18}$ \\
\hline 26 & 29.24 & Procyanidin B4 & se, sk & 577.1332 & 407 (93), 289 (73), 245 (59) & 577 & 152 & 289 & 577.1313 & -1.9 & $\mathrm{C}_{30} \mathrm{H}_{26} \mathrm{O}_{12}$ \\
\hline 27 & 29.85 & Procyanidin $\mathrm{B}^{*}$ & se, sk & 577.1331 & 407 (93), 289 (73), 245 (59) & 577 & 152 & 289 & 577.1311 & -2.0 & $\mathrm{C}_{30} \mathrm{H}_{26} \mathrm{O}_{12}$ \\
\hline 28 & 31.58 & (-)-Epicatechin ${ }^{*}$ & se, sk, pu & 289.0712 & 245 (100), 205 (60) & 289 & & & 289.0707 & -0.5 & $\mathrm{C}_{15} \mathrm{H}_{14} \mathrm{O}_{6}$ \\
\hline 29 & 32.42 & $\begin{array}{l}\text { (epi)catechin- } \\
\text { (epi)catechingallate I }\end{array}$ & se & 729.1476 & $577(26), 451(34), 407$ (96), $289(100)$ & 729 & & 289 & 729.1491 & 1.5 & $\mathrm{C}_{37} \mathrm{H}_{30} \mathrm{O}_{16}$ \\
\hline 30 & 33.26 & Procyanidin trimer III & se & 865.1959 & $\begin{array}{l}865(42), 695 \text { (49), } 577 \text { (52), } 407 \text { (70), } 289 \\
(100)\end{array}$ & 865 & 152 & 289 & 865.1933 & -2.6 & $\mathrm{C}_{45} \mathrm{H}_{38} \mathrm{O}_{18}$ \\
\hline 31 & 33.81 & $\begin{array}{l}\text { (epi)catechin- } \\
\text { (epi)catechingallate II }\end{array}$ & se & 729.1472 & $577(37), 407(100), 289(70)$ & 729 & 152 & 289 & 729.1483 & 1.1 & $\mathrm{C}_{37} \mathrm{H}_{30} \mathrm{O}_{16}$ \\
\hline 32 & 35.42 & $\begin{array}{l}\text { (epi)catechin- } \\
\text { (epi)catechingallate III }\end{array}$ & se & 729.1473 & 577 (43), 407 (100), 289 (94) & 729 & 152 & & 729.1485 & 1.2 & $\mathrm{C}_{37} \mathrm{H}_{30} \mathrm{O}_{16}$ \\
\hline 33 & 38.76 & Quercetin-3-O-rutinoside & sk & 609.1466 & 609 (67), $301(100)$ & 609 & 308 & 301 & 609.1471 & 0.5 & $\mathrm{C}_{27} \mathrm{H}_{30} \mathrm{O}_{16}$ \\
\hline 34 & 39.30 & Quercetin-3-O-glucuronide & sk & 477.0636 & $301(72), 151(100)$ & 477 & 176 & 301 & 477.0598 & -3.8 & $\mathrm{C}_{21} \mathrm{H}_{18} \mathrm{O}_{13}$ \\
\hline 35 & 39.44 & (-)-Epicatechin-3-O-gallate & se & 441.0866 & 289 (100), 271 (47), 169 (85), 125 (6) & 441 & & & 441.0905 & 3.9 & $\mathrm{C}_{22} \mathrm{H}_{18} \mathrm{O}_{10}$ \\
\hline 36 & 39.65 & Quercetin-3-O-glucoside ${ }^{*}$ & sk & 463.0857 & $301(67), 151(100)$ & 463 & 162 & 301 & 463.0833 & -2.4 & $\mathrm{C}_{21} \mathrm{H}_{20} \mathrm{O}_{12}$ \\
\hline 37 & 40.27 & $\begin{array}{l}\text { Dihydroquercetin-3-O- } \\
\text { rhamnoside }\end{array}$ & sk & 449.1109 & $303(100), 151(75)$ & 449 & & 303 & 449.1129 & 2.0 & $\mathrm{C}_{21} \mathrm{H}_{22} \mathrm{O}_{11}$ \\
\hline 38 & 40.52 & Dimer digallate & se & 881.1975 & 881 (25), 729 (93), 407 (100) & 881 & 152 & & 881.2016 & 4.1 & $\mathrm{C}_{45} \mathrm{H}_{38} \mathrm{O}_{19}$ \\
\hline 39 & 40.95 & Quercetin-3-O-pentoside & sk & 433.0731 & $301(100), 151(77)$ & 433 & & & 433.0692 & -3.9 & $\mathrm{C}_{20} \mathrm{H}_{18} \mathrm{O}_{11}$ \\
\hline 40 & 41.21 & Kaempferol-3-O-glucuronide & sk & 461.0770 & 285 (100), 257 (18), 229 (25) 135 (8) & 461 & 176 & & 461.0815 & 4.5 & $\mathrm{C}_{21} \mathrm{H}_{18} \mathrm{O}_{12}$ \\
\hline 41 & 41.40 & Kaempferol-3-O-glucoside & sk & 447.0972 & $447(30), 285(100)$ & 447 & 162 & 285 & 447.1012 & 4.0 & $\mathrm{C}_{21} \mathrm{H}_{20} \mathrm{O}_{11}$ \\
\hline 42 & 41.56 & trans-piceid $^{*}$ & sk & 389.1246 & 227 (100), 185 (17), $143(6)$ & 389 & 162 & 227 & 389.1251 & 0.5 & $\mathrm{C}_{20} \mathrm{H}_{22} \mathrm{O}_{8}$ \\
\hline 43 & 42.40 & trans-resveratrol $^{*}$ & sk & 227.0750 & $185(13), 143(100)$ & 227 & & & 227.0786 & 3.6 & $\mathrm{C}_{14} \mathrm{H}_{12} \mathrm{O}_{3}$ \\
\hline
\end{tabular}

Comparison with standard. sk, skin; pu, pulp; se, seeds; PIS, product ion scan, NL, neutral loss; PrI, precursor ion scan; [M-H] ${ }^{-}$mass found.

Obtained as theoretical mass - experimental mass. 


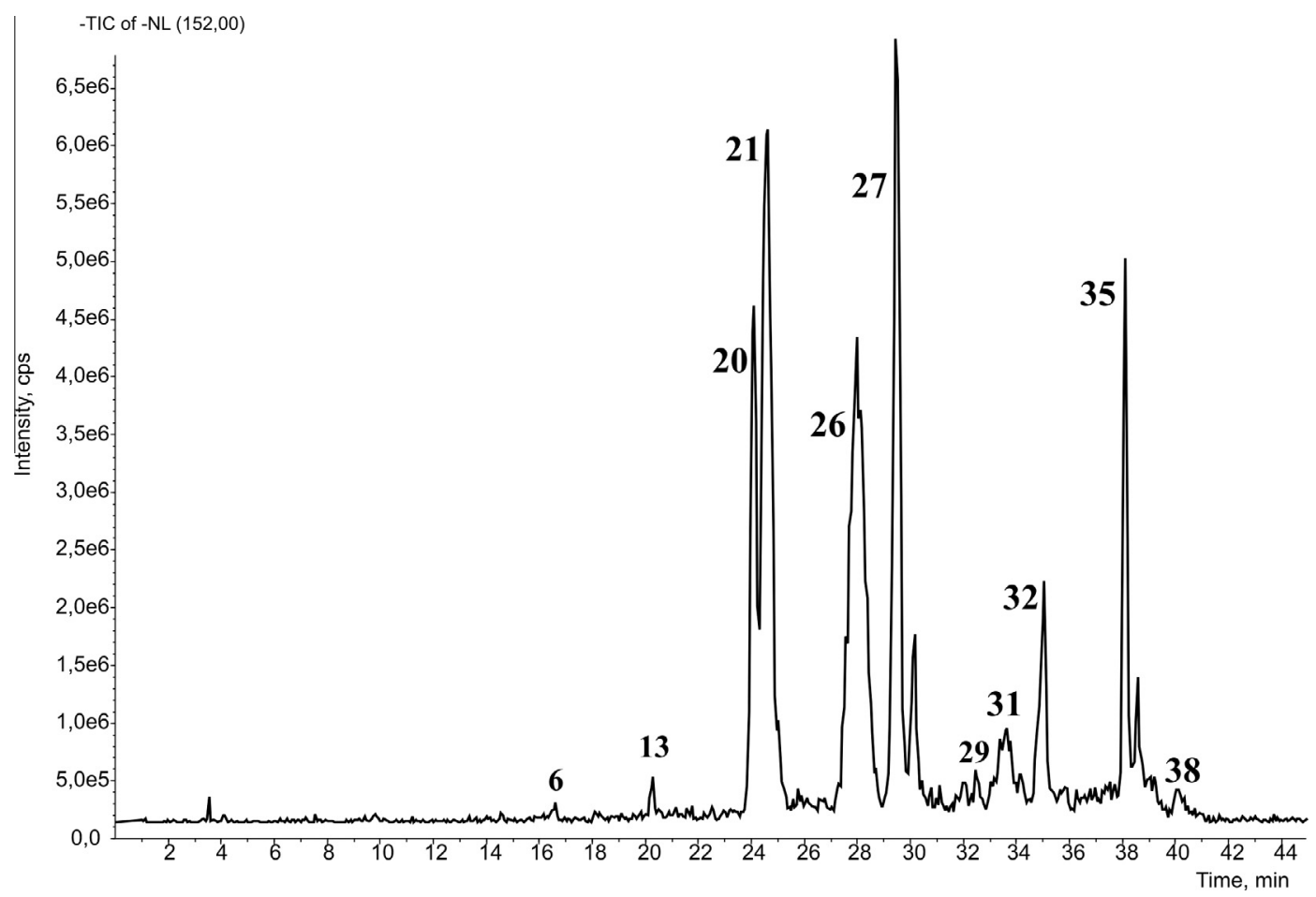

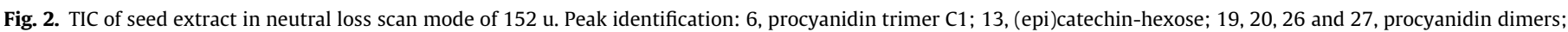
29, 31 and 32, (epi)catechin-(epi)catechingallate; 35, (-)-epicatechin-3-O-gallate; 38, dimer digallate.

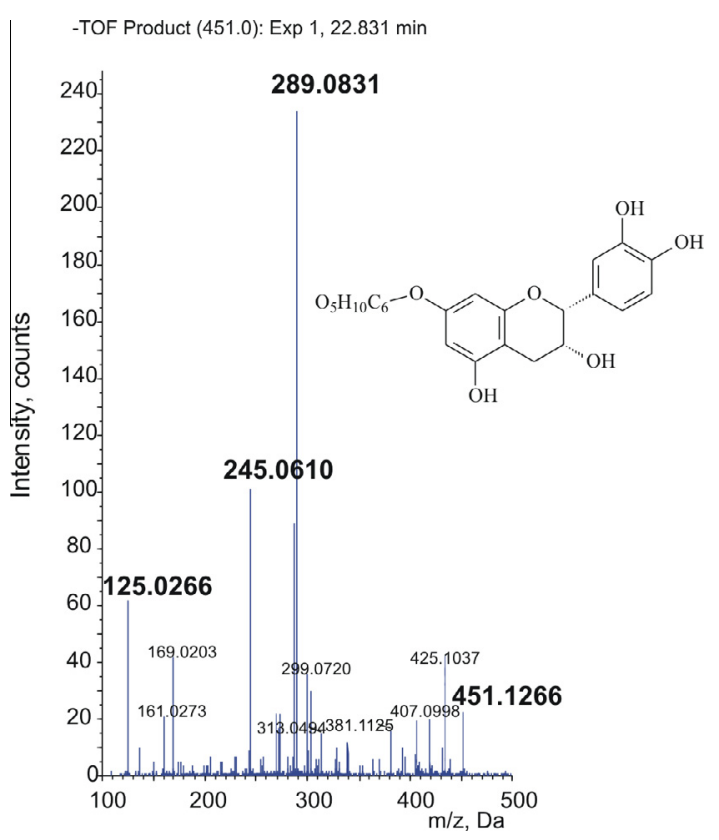

Fig. 3. The MS/MS product ion scan of $m / z 451$ (epi)catechin-hexose.

3.1.4.1. Flavanol hexosides. In Albariño seed, two flavan-3-ol hexoside isomers (peaks 13 and 16 ) at $\mathrm{m} / \mathrm{z} 451$ were tentatively identified as (+)-catechin or (-)-epicatechin-hexose. In PIS mode these flavanol hexosides showed a peak at $\mathrm{m} / \mathrm{z} 289$ due to the hexose moiety and the fragment at $\mathrm{m} / \mathrm{z} 245$ was attributed to (+)-catechin or (-)-epicatechin fragmentation (Fig. 2); the accurate mass measurements presented the same error for both compounds (2.1 $\mathrm{mDa}$ ). The NL of $162 \mathrm{u}$ and the PrI at $m / z$ 289, in LC-ESI-QqQ-MS, confirmed the tentative identification of flavanol hexosides.
To the best of our knowledge, this is the first time that flavanol monomer hexosides have been detected in grapes, although they have been reported in other plants. Two research groups previously described the presence of flavanol hexose in barley (Wolfgang \& Rudolf, 2002) and lentils (Dueñas, Sun, Hernández, Estrella, \& Spranger, 2003).

Catechins are associated with health benefits, but they are unstable during storage, processing and during gut transit (Zho et al., 2002). However, recent evidence suggests that catechin-glucoside is more stable (between $\mathrm{pH} 4$ and 8 ) than (+)-catechin. Raab et al., (2010) have shown that (+)-catechin-3'-O- $\beta$-D-glucopyranoside presents the greatest stability.

3.1.4.2. Flavan-3-ol galloylated derivatives. An ester derivative identified as (-)-epigallocatechin was found only in the skin. In fact the skin profile gave a peak at $m / z 305$, which represented a flavanolic unit; its confirmation was possible by comparing the chromatographic information with an authentic standard. As far as we know, flavan-3-ol galloylated derivatives have not been previously described in Albariño grape skin. Several monomeric and oligomeric flavanols linked to gallic acid were detected in the seed extract. Peak 9 showed an ion at $m / z 457$ which in PIS mode generated a preponderant fragment at $m / z 305$, probably produced by the loss of a galloyl group to epigallocatechin or gallocatechin. It was not possible to confirm the molecular structure of epigallocatechingallate or gallocatechin-gallate due to the lack of reference compounds. Three isomers of (epi)catechin-(epi)catechin-gallate, commonly known as dimer gallate $(m / z 729)$, were also tentatively identified in seeds with mass errors below $1.5 \mathrm{mDa}$. The PIS of $m / z 729$ generated an ion at $m / z 577$ corresponding to the loss of gallic acid, while the more intense fragment at $m / z 289$ was due to the loss of (+)-catechin-gallate or a (-)-epicatechin-gallate unit. For the dimer gallates, the PrI by LC-ESI-QqQ-MS at $m / z 289$ showed peaks at $m / z 729$, thus providing further useful information for checking characteristic phenolic compounds of grape seed. 
Another peak present only in the seed fraction at $m / z 441$ was identified as (-)-epicatechin-3-O-gallate as its retention time and mass spectra matched the standard. Moreover, the PIS showed two fragment ions resulting from the cleavage of the ester bond at $m / z 289$ for deprotonated (-)-epicatechin and at $m / z 169$ for a deprotonated gallic acid moiety. A prodelphynidin compound was also found in both seed and skin. LC-ESI-QqToF-MS analysis revealed the existence of a deprotonated molecule at $\mathrm{m} / \mathrm{z} 593$ and the PIS showed fragments at $m / z 423,305$ and 289, which confirmed the presence of (epi)gallocatechin-(epi)catechin. In addition, another ion was detected in the seed extract at $m / z 881$, which was tentatively identified as (epi)catechin-(epi)gallocatechin-(epi)catechin or (epi)gallocatechin-(epi)catechin-(epi)catechin due to another (epi)catechin linked to the molecular structure. The PIS experiment suggests that the more abundant fragment was at $m / z 729$, which corresponds to the gallate unit moiety (Lazarus, Adamson, Hammerstone, \& Schmitz, 1999). As depicted in Fig. 3, the total ion current of NL at $152 \mathrm{u}$ could also be used for a fast screening of flavan3-ol galloylated derivatives.

As described by Flamini (2003), dimer gallates were first identified in Niagara grapes. Other authors have confirmed that the grape seed phenolic profile is characterised by the presence of flavanol derivatives esterified with gallic acid and their occurrence can be considered typical of grape seeds (Santos-Buelga, Francis-Aricha, \& Escribano-Bailón, 2005; Rodríguez Montealegre et al., 2006).

\subsubsection{Flavonols}

In the skin fraction three flavonol-O-hexosides (peaks 36, 37 and 41), two-O-glucuronides (peaks 34 and 40), one -O-rutinoside (peak 33) and one -O-pentoside (39) were plausibly identified (Fig. 1 b and c). In this work, we found only - $\mathrm{O}$-glycoside derivatives arising from cleavage of the glycosidic bond and loss of the sugar moieties (Castillo-Muñoz, Gómez-Alonso, García-Romero, \& Hermosín-Gutiérrez, 2010). LC-ESI-QqToF-MS analysis of the skin phenolic extract revealed mass signals at $m / z 609,477$ and 463 , corresponding to deprotonated quercetin-3-O-rutinoside (also called rutin), quercetin-3-O-glucuronide and quercetin-3-O-glucoside (quercitrin), for peaks 33,34 and 36 , respectively. The deprotonated molecule at $\mathrm{m} / \mathrm{z} 609$ in PIS mode showed an intense fragment at $m / z 301$ due to the rutinoside moiety, and a fragment at $\mathrm{m} / z 151$ typical for quercetin. Similar $\mathrm{MS}^{2}$ experiments have been done for quercetin-3-O-glucuronide and quercetin-3-O-glucoside, showing the same fragment at $\mathrm{m} / \mathrm{z} 301$ for the glucuronide and glucoside moieties. The presence of the three quercetin derivatives was also checked by $\operatorname{PrI}$ at $\mathrm{m} / \mathrm{z} 301$ and by matching the retention time and mass spectra with data of available standard compounds. The presence of another quercetin derivative was detected by LC-ESI-QqToF-MS which showed a deprotonated molecular $[\mathrm{M}-\mathrm{H}]^{-}$at $m / z 433$ and the PIS showed a fragment at $m / z 301$. In this case, NL and PrI experiments did not yield any information due to the low intensity of the peak. This compound was tentatively assigned to quercetin-3-O-pentose and, as far as we know, this is the first time that this phenolic compound has been reported in Albariño grape skin. The chromatogram of the skin extract in QqToF-MS also showed a deprotonated molecule at $\mathrm{m} / \mathrm{z}$ 449 with an error of $2.0 \mathrm{mDa}$. The PIS of $\mathrm{m} / z 449$ gave a product ion at $m / z 303$, which suggested the probable presence of diihydroquercetin-3-O-rhamnoside, as described by Masa and Vilanova (2008) for Albariño skin. Analysis of the skin phenolic profile also revealed the presence of two ions at $\mathrm{m} / \mathrm{z} 461$ and 447 corresponding to kaempferol-3-O-glucuronide and kaempferol-3$O$-glucoside, respectively. The PIS in LC-ESI-QqToF-MS bore out these results, in both cases showing a preponderant fragment at $m / z$ 285. Additionally, as described above, NL and PrI experiments characterised kaempferol-3-O-glucuronide and kaempferol-3-O- glucoside, which were corroborated by comparison with the standard compounds (Table 1 ).

Flavonoid-C-glycosides, which show a different fragmentation pattern from -O-glycosides (Sanchez-Rabaneda et al., 2003; Han et al., 2008), were not detected in the Albariño grape.

\subsubsection{Stilbenes}

Stilbenes were eluted in the final part of the Albariño skin chromatogram. Peaks 42 and 43 showed deprotonated molecules at $m / z$ 389 and $227[\mathrm{M}-\mathrm{H}]^{-}$, and were identified as trans-piceid and transresveratrol, respectively. Both stilbenes were corroborated by comparison with the reference compounds. The presence of resveratrol and its glucoside in red as well as white grapes has been ascribed to ultraviolet irradiation or stress, especially plant interaction with pathogens (Romero-Pérez, Lamuela-Raventós, Andreés-Lacueva, \& de la Torre-Boronat, 2001).

Furthermore, as reported by Lamuela-Raventos, Romero-Pérez, Waterhouse and de la Torre-Boronat, (1995), trans isomers are transformed to the cis forms when grapes are exposed to UV radiation. Probably due to the extraction procedure, which was performed in a dark room with a red safety light, we did not detect cis isomer forms in Albariño skin. Stilbenes have been extensively studied as critical contributors to the health benefits of grapes and wine (Lamuela-Raventós \& Waterhouse, 1999).

\subsection{A nitrogen compound with phenolic structure: L-tryptophan}

A nitrogen compound identified as L-tryptophan (peak 11) was found in all the anatomical parts of Albariño grapes. In LC-ESIQqToF-MS, this peak showed an ion at $m / z 203$ and the PIS revealed two ions at $m / z 116$ and 142 . The compound identity was confirmed by comparing its mass spectra with those of an authentic standard. L-tryptophan can be present in white must and is ascribed to the metabolic pathway of 2-aminoacetophenone, a causal agent of an 'untypical ageing off-flavour' in wine. In another study, Mattivi, Vrhovšek, and Versini, (1999) found levels of L-tryptophan in Chardonnay musts and wines ranging between 62 and $417 \mu \mathrm{g}$ $\mathrm{L}^{-1}$. (Mattivi et al., 1999).

\subsection{Quantification of phenolic compounds found in skin, pulp and seed}

The most abundant class of phenols found in Albariño grape berries were the monomeric and oligomeric form of flavan-3-ols, which were present in hypodermal layers of skin and in the soft parenchyma of the seed. The total content of flavanols was $611 \mathrm{mg} * 100 \mathrm{~g}^{-1}$ of fresh matter (Table 2), while the compounds at the highest concentration were (+)-catechin and (-)epicatechin.

Albariño grape skin exhibited a predominance of flavanols and flavonols but a considerable amount of hydroxycinnamates was also found. The major hydroxycinnamic acid present was cis-coutaric acid (see Table 2), followed by caftaric isomers. Flavonols were always found in glycoside form, principally as 3-glucosides; small amounts of rutinoside and glucuronide flavonols were also detected. The content of flavonols ranged between 0.39 and $12.4 \mathrm{mg} * 100 \mathrm{~g}^{-1}$ of fresh matter for quercetin-3-O-rutinoside and quercetin-3-O-glucoside, respectively. As reported by Downey, Harvey, and Robinson (2004), the flavonol content cannot be considered as characteristic of a grape cultivar because the flavonol concentration is strongly affected by the degree of illumination of the grape cluster (Downey et al., 2004).

The phenolic content in the pulp was very low. Hydroxycinnamic acids were the most representative compounds, with a total content of about $1.63 \mathrm{mg} * 100 \mathrm{~g}^{-1}$ of fresh matter. Small amounts of catechin, epicatechin and procyanidin B3 were also found. 
Table 2

Content of phenolic compounds in the different anatomical parts of Albariño grape ( $\mathrm{mg}^{*} 100 \mathrm{~g}^{-1}$ of fresh matter). Mean values ( \pm standard errors); nd, not detected.

\begin{tabular}{|c|c|c|c|}
\hline Compounds & Skin & Pulp & Seed \\
\hline Gallic acid & 1.19 & nd & 1.92 \\
\hline Gallic acid hexose I & nd & nd & 0.79 \\
\hline Gallic acid dihexose & 1.13 & nd & 1.25 \\
\hline Protocatechuic acid-O-hexoside & nd & nd & 0.45 \\
\hline Gallic acid hexose II & nd & nd & 1.36 \\
\hline Protocatechuic acid-O-hexoside & nd & nd & 1.54 \\
\hline Hydroxybenzoic acids & $2.32 \pm 0.1$ & nd & $7.31 \pm 0.3$ \\
\hline cis-caftaric acid & 2.53 & 0.11 & nd \\
\hline trans-caftaric acid & 4.04 & 0.37 & nd \\
\hline cis-coutaric acid & 6.23 & nd & nd \\
\hline trans-coutaric acid & 0.27 & nd & nd \\
\hline Coumaric acid- $O$-hexoside & 2.27 & 1.03 & nd \\
\hline Fertaric acid & 1.68 & 0.12 & nd \\
\hline$p$-coumaric acid & 1.96 & nd & nd \\
\hline Hydroxycinnamic acids & $18.98 \pm 0.8$ & $1.63 \pm 0.1$ & nd \\
\hline (epi)gallocatechin-(epi)catechin & nd & nd & 3.58 \\
\hline Procyanidin trimer $\mathrm{C} 1$ & nd & nd & 12.65 \\
\hline (epi)gallocatechin-3-gallate & nd & nd & 1.54 \\
\hline Epigallocatechin & 2.09 & nd & nd \\
\hline (epi)catechin-3-hexose & nd & nd & 1.49 \\
\hline (epi)catechin-3-hexose & nd & nd & 3.52 \\
\hline Procyanidin B3 & nd & 0.57 & 44.65 \\
\hline Procyanidin B1 & nd & nd & 3.09 \\
\hline$(+)$-catechin & 11.45 & 0.55 & 106.5 \\
\hline Procyanidin trimer I & nd & nd & 31.43 \\
\hline Procyanidin trimer II & nd & nd & 18.54 \\
\hline Procyanidin B4 & 8.04 & nd & 58.39 \\
\hline Procyanidin B2 & 8.65 & nd & 64.53 \\
\hline (-)-epicatechin & 2.67 & 0.23 & 77.51 \\
\hline (epi)catechin-(epi)catechingallate I & nd & nd & 26.76 \\
\hline Procyanidin trimer III & nd & nd & 13.54 \\
\hline (epi)catechin-(epi)catechingallate II & nd & nd & 23.73 \\
\hline (epi)catechin-(epi)catechingallate III & nd & nd & 21.43 \\
\hline (-)-epicatechin-3-O-gallate & nd & nd & 76.54 \\
\hline Dimer digallate & nd & nd & 21.43 \\
\hline Flavanols & $32.9 \pm 2.7$ & $1.35 \pm 0.1$ & $610.8 \pm 35.8$ \\
\hline Quercetin-3-O-rutinoside & 0.42 & nd & nd \\
\hline Quercetin-3-O-glucuronide & 0.98 & nd & nd \\
\hline Quercetin-3-O-glucoside & 12.43 & nd & nd \\
\hline dihydroquercetin-3-O-rhamnoside & 5.65 & nd & nd \\
\hline Quercetin-3-O-pentoside & 0.23 & nd & nd \\
\hline Kaempferol-3-O-glucuronide & 3.21 & nd & nd \\
\hline Kaempferol-3-O-glucoside & 8.43 & nd & nd \\
\hline flavonols & $31.45 \pm 1.6$ & nd & nd \\
\hline trans-piceid & 6.93 & nd & nd \\
\hline trans-resveratrol & 1.43 & nd & nd \\
\hline Stilbenes & $8.36 \pm 0.4$ & nd & nd \\
\hline Total & $94.21 \pm 5.1$ & $2.98 \pm 0.2$ & $618.1 \pm 36.1$ \\
\hline
\end{tabular}

As described by other authors, the concentration of phenolics and their profile in grapes, depends on the grapevine variety as well as intrinsic factors such as genetics and extrinsic aspects linked to viticulture and the environment. The degree of ripeness and berry size are also influential (Rodríguez Montealegre et al. 2006). In accordance with previous papers, the results presented in this study demonstrate that grape berries generally present a very high polyphenolic content, which contributes to their value as an agricultural crop (Rodríguez Montealegre et al., 2006; Dietmar et al., 2004).

\section{Conclusions}

Using a combination of spectrometric techniques we were able to identify up to 43 compounds, two of which, (+)-catechin or (-)epicatechin hexosides, as far as we know, have never been reported before in the grape berry. The QqToF-MS was very useful for its combination of high sensitivity, high resolution and high mass accuracy, also allowing the characterisation of deprotonated molecules from PIS experiments in HRMS mode. Good fits were ob- tained for all investigated ions, with errors ranging from 0.2 to $4.5 \mathrm{mDa}$. The QqQ system was effective for obtaining information about the phenolic composition of grapes through NL and PrI experiments that allowed a first screening of families of compounds. In particular, an NL of $152 \mathrm{u}$ was found to be helpful for a rapid screening of procyanidin dimers and trimers and gallate flavanols.

A wide range of phenolic compounds was found diversely distributed in every part of the Albariño grape berries but mainly in the skin and seed. Thus, this investigation resulted in an exhaustive characterisation of the phenolic profile of the different anatomical parts of the Albariño grape, and provides useful information for selecting suitable by-products for the extraction of potential health-promoting compounds.

\section{Acknowledgements}

The authors express their gratitude to CENIT-DEMETER FBG 305273, to the following companies: Bodegas Roda S. A., Bodegas Martin Codax A. U., Miguel Torres S. A., Ecovitis S. L., Bodega Matarromera S. L., Pago De Carraovejas S. A., Lallemand Bio S. L., Productos Agrovin S. A., Avanzare Inno Tecnologica S. L., Bodegas Licinia S. L., Dominio De La Vega S. L., Solfranc Tecnologías S. L., Gramona S. A., Juve I Camps S. A., Castell D’Encus, Ferrer Bobet S. L., Laffort España S. A., Dolmar Distribuidora Enológica S. L., Bodegas Protos Ribera Duero De Peñafiel S. L., Intranox S. L., Toneleria Magreñan S. L., Tecnología y Difusion Ibérica S. L., Union de Cosecheros De Labastida S. Coop., Hera Amasa S. A., Aecork (Asociación Patronal). S. Arranz and A. Tresserra-Rimbau thanks the Sara Borrell program supported by the Instituto de Salud Carlos III, Spain.

\section{References}

Adam, D. O. (2006). Phenolics and ripening in grape berries. American Journal of Enology and Viticulture, 57, 249-256.

Betés-Saura, C., Andrés-Lacueva, C., \& Lamuela-Raventós, R. M. (1996). Phenolics in white free run juices and wines from penedés by high-performance liquid chromatography: Changes during vinification. Journal of Agriculture and Food Chemistry, 44, 3040-3046.

Castillo-Muñoz, N., Gómez-Alonso, S., García-Romero, E., \& Hermosín-Gutiérrez, I. J. (2010). Flavonol profiles of Vitis vinifera white grape cultivars. Journal of Food Composition and Analysis, 23, 699-705.

Cavaliere, C., Foglia, P., Gubbiotti, R., Sacchetti, P., Samperi, R., \& Laganà, A. (2008), Rapid-resolution liquid chromatography/mass spectrometry for determination and quantitation of polyphenols in grape berries. Rapid Communications Mass Spectrometry, 22, 3089-3099.

Diéguez, S. C., Lois, L. C., Gómez, E. F., \& de la Peña, M. G. (2003). Aromatic composition of the Vitis vinifera grape Albariño. Lebensm.-Wiss. u.-Technol, 36, 585-590.

Dietmar, K., Achim, C., Reinhold, C., \& Schieber, A. (2004). Polyphenol screening of pomace from red and white grape varieties (Vitis vinifera L.) by HPLC-DAD-MS/ MS. Journal Agricultural and Food Chemistry, 52, 4360-4367.

Downey, M. O., Harvey, J. S., \& Robinson, S. P. (2004). The effect of bunch shading on berry development and flavonoid accumulation in Shiraz grapes. Australian Journal of Grape and Wine Research, 10, 55-73.

Dueñas, M., Sun, B., Hernández, T., Estrella, I., \& Spranger, M. I. (2003). Proanthocyanidin composition in the seed coat of lentils (Lens culinaris L.). Journal of Agriculture and Food Chemistry, 51, 7999-8004.

Flamini, A. (2003). Mass spectrometry in grape and wine chemistry. Part I: Polyphenols. Mass Spectrometry Reviews, 22, 218-250.

Gu, L., Kelm, M. A., Hammerstone, J. F., Zhang, Z., Beecher, G., Holden, J., et al. (2003). Liquid chromatographic/electrospray ionization mass spectrometric studies of proanthocyanidins in foods. Journal of Mass Spectrometry, 38, 1272-1280.

Han, J., Ye, M., Qiao, X., Xu, M., Wang, B. R., \& Guo, D. A. (2008). Characterization of phenolic compounds in the Chinese herbal drug Artemisia annua by liquid chromatography coupled to electrospray ionization mass spectrometry. Journal of Pharmaceutical and Biomedical Analysis, 47, 516-525.

Hayasaka, Y., Waters, E. J., Cheynier, V., Herderich, M. J., \& Vidal, S. (2003). Characterization of proantocyanidins in grape seeds using electrospray mass spectrometry. Rapid Communications Mass Spectrometry, 17, 9-16.

Kroon, P. A., \& Williamson, G. (1999). Hydroxycinnamates in plants and wood. Current and future perspectives. Journal Science Food Agriculture, 79, 355-361.

Justesen, U., Knuthsen, P., \& Leth, T. (1998). Quantitative analysis of flavonols, flavones, and flavanones in fruits, vegetables and beverages by highperformance liquid chromatography with photo-diode array and mass spectrometric detection. Journal of Chromatography A, 799, 101-110. 
Lazarus, S. A., Adamson, G. E., Hammerstone, J. F., \& Schmitz, H. H. (1999). Highperformance liquid chromatography/mass spectrometry analysis of proanthocyanidins in foods and beverages. Journal of Agriculture and Food Chemistry, 47, 3693-3701.

Lamuela-Raventos, R. M., Romero-Pérez, A. I. Waterhouse, A. L. \& de la TorreBoronat, M. C. (1995). Direct HPLC analysis of cis- and trans-resveratrol and piceid isomers in Spanish red Vitis vinifera wines. Journal of Agriculture and Food Chemistry, 43, 281-283.

Lamuela Raventós, R. M., \& Waterhouse, A. L. (1999). Resveratrol and piceid in wine. In L. Parker (Ed.), Oxidants and antioxidants, methods in enzymology (pp. 184-190). San Diego: Academic Press.

Masa, A., \& Vilanova, M. (2008). Flavonoid and aromatic characterisation of cv. Albarín blanco (Vitis vinifera L.). Food Chemistry, 107, 273-281.

Masa, A., Vilanova, M., \& Pomar, F. (2007). Varietal differences among the flavonoid profiles of white grape cultivars studied by high-performance liquid chromatography. Journal of Chromatography A, 1164, 291-297.

Mattivi, F., Vrhovšek, U., \& Versini, G. (1999). Determination of indole-3-acetic acid, tryptophan and other indoles in must and wine by high-performance liquid chromatography with fluorescence detection. Journal of Chromatography A, 855, 227-235.

Monagas, M., Suárez, R., Gómez-Cordovés, C., \& Bartolomé, B. (2005). Simultaneous determination of nonanthocyanin phenolic compounds in red wines by HPLCDAD/ESI-MS. American Journal of Enology and Viticulture, 56, 139-147.

Raab, T., Barron, D., Arce Vera, F., Crespy, V., Oliveira, M., \& Williamson, G. (2010). Catechin glucosides: Occurrence, synthesis and stability. Journal of Agriculture and Food Chemistry, 58, 2138-2149.

Rodríguez-Bernaldo de Quirós, A., Lage-Yusty, M. A., \& López-Hernández, J. (2009). HPLC-analysis of polyphenolic compounds in Spanish white wines and determination of their antioxidant activity by radical scavenging assay. Food Research International, 42, 1018-1022.

Rodríguez Montealegre, R., Romero Peces, R., Chacón Vozmediano, J. L., Martínez Gascueña, J., \& García Romero, E. J. (2006). Phenolic compounds in skins and seeds of ten grape Vitis Vinifera varieties grown in a warm climate. Journal Composition and Analysis, 19, 687-693.

Romero-Pérez, A., Ibern-Gómes, M., Lamuela-Raventós, R. M., \& de la Torre-Boronat, M. C. (1999). Piceid, the major resveratrol derivative in grape juice. Journal of Agriculture and Food Chemistry, 47, 1533-1536.

Romero-Pérez, A., Lamuela-Raventós, R. M., Andreés-Lacueva, C., \& de la TorreBoronat, M. C. (2001). Method for the quantitative extraction of resveratrol and piceid isomers in grape berry skins. Effect of powdery mildew on the stilbene content. Journal of Agriculture and Food Chemistry, 49, 210-215.

Sanchez-Rabaneda, F., Jauregui, O., Casals, I., Andres-Lacueva, C., Izquierdo-Pulido, M., \& Lamuela-Raventós, R. M. (2003). Liquid chromatographic/electrospray ionization tandem mass spectrometric study of the phenolic composition of cocoa (Theobroma cacao). Journal of Mass Spectrometry, 38, 35-42.

Sánchez-Rabaneda, F., Jáuregui, O., Lamuela-Raventós, R. M., Viladomat, F., Bastida, J., \& Codina, C. (2004). Qualitative analysis of phenolic compounds in apple pomace using liquid chromatography coupled to mass spectrometry in tandem mode. Journal of Mass Spectrometry, 18, 553-563.
Santos-Buelga, C., Francis-Aricha, E. M., \& Escribano-Bailón, M. T. (2005) Comparative flavan-3-ol composition of seeds from different grape varieties. Food Chemistry, 53, 197-201.

Sun, W., \& Miller, J. M. (2003). Tandem mass spectrometry of the B-type procyanidins in wine and B-type dehydrodicatechins in an autoxidation mixture of (+)-catechin and (-)-epicatechin. Journal of Mass Spectrometry, 38, 438-446.

Vallverdu-Queralt, A., Jáuregui, O., Medina-Remón, A., Andres-Lacuéva, C., \& Lamuela-Raventós, M. (2010). Improved characterization of tomato polyphenols using liquid chromatography/electrospray ionization linear ion trap quadrupole Orbitrap mass spectrometry and liquid chromatography/ electrospray ionization tandem mass spectrometry. Rapid Communications Mass Spectrometry, 24, 2986-2992.

Vallverdú-Queralt, A., Medina-Remón, A., Martínez-Huélamo, M., Jáuregui, O. Andrés-Lacueva, C., \& Lamuela-Raventós, R. M. (2011a). Phenolic profile and hydrophilic antioxidant capacity as chemotaxonomic markers of tomato varieties. Journal of Agricultural and Food Chemistry, 59, 3994-4001.

Vallverdú-Queralt, A., Jáuregui, O., Di Lecce, G., Andrés-Lacueva, C., \& LamuelaRaventós, R. M. (2011b). Screening of the polyphenol content of tomato-based products through accurate-mass spectrometry (HPLC-ESI-QTOF). Food Chemistry, 129, 877-883.

Vidal, S., Francis, L., Guyot, S., Marnet, N., Kwiatkowski, M., Gawel, R., et al. (2003) The mouth-feel properties of grape and apple proanthocyanidins in a wine-like medium. Journal of the Science of Food and Agriculture, 83, 564-573.

Vilanova, M., Genisheva, Z., Masa, A., \& Oliveira, J. M. (2010). Correlation between volatile composition and sensory properties in Spanish Albariño wines. Microchemical Journal, 95, 240-246.

Vrhovšek, U. (1998). Extraction of hydroxycinnamoyltartaric acids from berries of different grape varieties. Journal of Agriculture and Food Chemistry, 46, 4203-4208.

Waterhouse, A. L., Ignelzi, S., \& Shirley, J. R. (2000). A comparison of methods for quantifying oligomeric proanthocyanidins from grape seed extracts. American Journal of Enology and Viticulture, 51, 383-389.

Wolfgang, F., \& Rudolf, G. (2002). Identification of a new flavanol glucoside from barley (Hordeum vulgare L.) and malt. European Food Research and Technology, 214, 388-393.

Zhao, J., Pang, Y., \& Dixon, R. A. (2010). The mysteries of proanthocyanidin transport and polymerization. Plant Physiology, 153, 437-443.

Zhou, Y., Xu, G., Choi, F. K., Dingc, F., Bin Han, Q., Zheng Song, J., et al. (2009) Qualitative and quantitative analysis of diterpenoids in Salvia species by liquid chromatography coupled with electrospray ionization quadrupole time-offlight tandem mass spectrometry. Journal of Chromatography A, 1216 4847-4858.

Zho, Q. Y., Holt, R. R., Lazarus, S. A., Ensunsa, J. L., Hammerstone, J. F., Schimitz, H. H et al. (2002). Stability of the flavan-3-ols epicatechin and catechin and related dimeric procyanidins derived from cocoa. Journal of Agriculture and Food Chemistry, 50, 1700-1705. 